\title{
Erratum to: Rehabilitative Surgery: A Comprehensive Text for an Emerging Field
}

\author{
Andrew I. Elkwood, Matthew Kaufman, \\ and Lisa F. Schneider
}

Erratum to

A.I. Elkwood et al. (eds.), Rehabilitative Surgery, DOI 10.1007/978-3-319-41406-5

In the original version of front matter, Foreword in Book Front matter was missing.

In the original version for chapter 20, the author name Matthew Pontell, MD, Department of Surgery, Drexel University College of Medicine, Philadelphia, PA, USA was missing which has been included.

The above mentioned corrections also updated in Table of Contents.

The updated online version of the original book can be found under DOI 10.1007/978-3-319-41406-5 